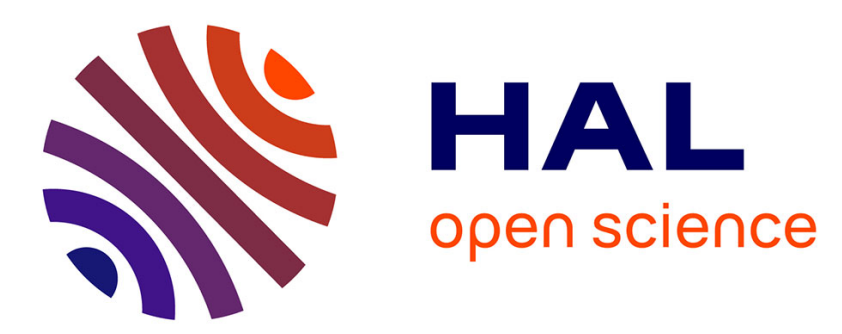

\title{
Observability analysis and sensor location study for structured linear systems in descriptor form with unknown inputs
}

Taha Boukhobza, Frédéric Hamelin

\section{- To cite this version:}

Taha Boukhobza, Frédéric Hamelin. Observability analysis and sensor location study for structured linear systems in descriptor form with unknown inputs. Automatica, 2011, 47 (12), pp.2678-2683. 10.1016/j.automatica.2011.08.048 . hal-00640470

\section{HAL Id: hal-00640470 https://hal.science/hal-00640470}

Submitted on 12 Nov 2011

HAL is a multi-disciplinary open access archive for the deposit and dissemination of scientific research documents, whether they are published or not. The documents may come from teaching and research institutions in France or abroad, or from public or private research centers.
L'archive ouverte pluridisciplinaire HAL, est destinée au dépôt et à la diffusion de documents scientifiques de niveau recherche, publiés ou non, émanant des établissements d'enseignement et de recherche français ou étrangers, des laboratoires publics ou privés. 


\title{
Observability analysis and sensor location study for structured linear systems in descriptor form with unknown inputs
}

\author{
T. Boukhobza and F. Hamelin \\ Centre de Recherche en Automatique de Nancy (CRAN), Nancy-University, CNRS UMR 7039 \\ email: taha.boukhobza@cran.uhp-nancy.fr
}

\begin{abstract}
This paper deals with the state and input right-hand side observability and the sensor location problem to ensure this property for structured linear systems in descriptor form. The proposed method is based on a graph-theoretic approach. It is applicable to a large class of descriptor systems including regular and nonregular systems even if they are underdetermined, overdetermined or square. After defining a bipartite graph which represents such systems, we deduce some graphic necessary and sufficient conditions to achieve the right-hand side observability of the state and the input. Next, we propose a sensor placement procedure to recover this property. More precisely, we provide the minimal number of the required sensors and either their pertinent location or a necessary and sufficient condition to check if a given sensor configuration is adequate or not to ensure state and input right-hand side observability. Our approach uses algorithms with polynomial complexity orders and assumes only the knowledge of the system's structure and so is suited for large scale systems.
\end{abstract}

Key words: Descriptor systems, right-hand side observability, sensor location, graph theory.

\section{Introduction}

Descriptor systems result from a convenient and natural modelling process (Luenberger, 1977; Lewis, 1992) and their applications can be found in various fields (Müller, 2000) such as robotics, electrical circuit networks, biologic and economic systems. Many of the structural properties of descriptor systems are revisited in (Aplevich, 1991; Dai, 1989; Yip and Sincovec, 1981). Among these properties, the observability analysis of the state and the unknown input of descriptor systems is a great interest mainly in the design of control laws, fault detection and isolation schemes, fault tolerant control strategies, supervision proposals and so on. In most cases, the studies concerning the observability of descriptor systems deal with algebraic and geometric tools, which assume the exact knowledge of the state space matrices characterizing the model of the system. In many modelling problems, these matrices have a number of fixed zero entries, determined by the physical structure, while the remaining entries are not exactly known. To study the properties of these systems in spite of the poor knowledge we have of them, the idea is that we only keep the zero/nonzero entries in the matrices of their state-space representation. Many interesting works on these models, called structured models, are related to the graph-theoretic approach and aim to analyse properties such as controllability, observability ...(Dion et al., 2003). It results from these works that the graph-theoretic approach provides quite simple and elegant analysis tool. Concerning descriptor systems, the studied structured form is

$$
\Sigma_{\Lambda}:\left\{\begin{aligned}
& E^{\lambda} \dot{x}=A^{\lambda} x+B^{\lambda} u \\
& y=C^{\lambda} x+D^{\lambda} u \\
& z \stackrel{\text { def }}{=} E^{\lambda} x
\end{aligned}\right.
$$

where $x \in \mathbb{R}^{n}, u \in \mathbb{R}^{m}$ and $y \in \mathbb{R}^{p}$ are respectively the state vector, the unknown input vector and the output vector. $E^{\lambda} \in \mathbb{R}^{q \times n}, A^{\lambda} \in \mathbb{R}^{q \times n}, B^{\lambda} \in \mathbb{R}^{q \times m}, C^{\lambda} \in \mathbb{R}^{p \times n}$ and $D^{\lambda} \in \mathbb{R}^{p \times m}$ represent matrices which elements are either fixed to zero or assumed to be nonzero parameters noted $\lambda_{i}$. The vector of these parameters is $\Lambda=\left(\lambda_{1}, \lambda_{2}, \ldots, \lambda_{h}\right)^{T}$ and it is assumed that $\Lambda$ can take any value in $\mathbb{R}^{h}$. If all parameters $\lambda_{i}$ are numerically fixed, we obtain a so-called admissible realization of $\Sigma_{\Lambda}$. We say that a property is true generically for $\Sigma_{\Lambda}$ if it is true for almost all its realizations or equivalently for almost all parameters $\lambda_{i}$. Here, "for almost all its realizations" is to be understood as "for all parameter values $\left(\Lambda \in \mathbb{R}^{h}\right)$ except for those in some proper algebraic variety in the parameter space".

Among the studies analysing structured systems in descriptor form by means of a graph-theoretic approach, we can cite (Reinschke and Wiedemann, 1997; Wiedemann, 1999; Murota, 1987; van der Woude, 2002; Boukhobza et al., 2006). In (Murota, 1987), the authors use bipartite graphs to characterize structural controllability, observability of descriptor systems. However, they make some assumptions about the studied systems which must be generically regular i.e. matrices $E^{\lambda}$ and $A^{\lambda}$ have to be square $(q=n)$ and generically $\operatorname{det}\left[s E^{\lambda}-A^{\lambda}\right] \not \equiv 0$. In (Reinschke and Wiedemann, 1997), the authors use digraphs to characterize structural controllability of descriptor systems which 
must be generically regular and satisfy another constraint: no main diagonal element of $\left[s E^{\lambda}-A^{\lambda}\right]$ equals zero. In (Wiedemann, 1999), structural properties of regular descriptor systems, including the observability, are studied by assuming also that there is no zero element in the main diagonal of $\left[s E^{\lambda}-A^{\lambda}\right]$. More recently, (van der Woude, 2002) studies the generic number of invariant zeros of a structured regular descriptor system. Many descriptor systems do not satisfy all these assumptions like the underdetermined ones which are very helpful to represent systems with unknown inputs. This fact motivates the works presented in (Boukhobza et al., 2006), where necessary and sufficient conditions for the total or partial state observability of possibly non-regular descriptor systems are given using a digraph. Unfortunately, to check these conditions, a recursive algorithm with an exponential complexity order must be used. In this context, the aim of this paper is, first, to extend the works of (Murota, 1987) to establish the right-hand side observability for as well regular and non-regular, which can be possibly non-square, descriptor systems with unknown inputs, but contrary to (Boukhobza et al., 2006) by the use of algorithms having polynomial complexity orders. In a second part of the paper, using also a graph-theoretic approach, we study the number and the location of the additional sensors which are necessary and useful to recover the right-hand side input and state observability conditions. We answer to the following question: when a descriptor system is not input and state right-hand side observable, where must we place a minimal number of additional sensors to recover this observability property? At this aim, we give the necessary and sufficient number of additional sensors we must add. All the proposed results are based on algorithms with polynomial complexity order. This may be an important criterion when we deal with large scale systems. Moreover, since we consider structured systems, our approach can be used during a conception stage.

The paper is organized as follows: after section 2, which is devoted to the problem formulation, a bipartite graph representation of structured systems is given in section 3. Our main results are presented in Section 4. A brief conclusion ends the paper.

\section{Problem statement}

First of all, let us precise the assumptions made on the system and the type of observability we study. Obviously, $u(t)$ and $E x\left(0_{-}\right)$are assumed to be admissible and are such that system $\Sigma_{\Lambda}$ is solvable (Yip and Sincovec, 1981; Geerts, 1993). Moreover, without loss of generality, we only assume that $E^{\lambda}$ does not have any row identically zero.

Note that no assumption is done on the rank of $\left[s E^{\lambda}-A^{\lambda}\right]$ neither on its main diagonal. Therefore, contrary to the works which assume the regularity of the system, our approach is adapted to analyse the state and also the input observability (Hou and Patton, 1998) of systems with unknown inputs since we do not made any hypothesis on the regularity of $\Sigma_{\Lambda}$. The kind of observability we study is related to the right-hand side observability (Hou and Müller, 1999a). Hereafter, we recall the definition of the right-hand side observability. This definition extends the one given in (Hou and Müller, 1999a) for non-structured descriptor systems:

Definition 1 Structured system $\Sigma_{\Lambda}$ is generically state and input right-hand side observable, if, for almost all the realizations of structured system $\Sigma_{\Lambda}, y(t)=0$ for $t \geq 0$ implies $x\left(0_{+}\right)=0$ and $u\left(0_{+}\right)=0$.

It is proved in (Hou and Müller, 1999b), that it ensures the existence of a generalized observer which allows to reconstruct the state and the input. In fact, $\Sigma_{\Lambda}$ is state and input right-hand side observable iff for $t>0$, state $x(t)$ and input $u(t)$ can be uniquely expressed using the measurements and their derivatives (Müller and Hou, 1993; Hou and Müller, 1999b).

To summarize, considering a structured system in descriptor form $\Sigma_{\Lambda}$, we propose first to answer the question whether or not state $x(t)$ and input $u(t)$ are generically right-hand side observable. The second and more original part of this paper is dedicated to the location of additional sensors to recover the right-hand side observability conditions. More precisely, starting from a system which is not generically state and input right-hand side observable, we propose a systematic procedure for additional sensor placement in order to recover the generic state and input right-hand side observability.

\section{Graphic representation of a structured descriptor system}

We associate to structured system $\Sigma_{\Lambda}$ a bipartite graph noted $B\left(\Sigma_{\Lambda}\right)=\left(\mathbf{V}^{+}, \mathbf{V}^{-}, W\right)$, where $\mathbf{V}^{+}$and $\mathbf{V}^{-}$are two disjoint vertex subsets and $W$ is the edge set. The vertices are associated to the internal state, the input and the output components of $\Sigma_{\Lambda}$ and the edges represent links between these variables. More precisely, $\mathbf{V}^{+}=\mathbf{X} \cup \mathbf{U}$ and $\mathbf{V}^{-}=$ $\mathbf{Y} \cup \mathbf{Z}$, with $\mathbf{X}=\left\{\mathbf{x}_{\mathbf{1}}, \mathbf{x}_{\mathbf{2}}, \ldots, \mathbf{x}_{\mathbf{n}}\right\}, \mathbf{U}=\left\{\mathbf{u}_{1}, \mathbf{u}_{2}, \ldots, \mathbf{u}_{\mathbf{m}}\right\}$, $\mathbf{Z}=\left\{\mathbf{z}_{1}, \mathbf{z}_{2}, \ldots, \mathbf{z}_{\mathbf{q}}\right\}, \mathbf{Y}=\left\{\mathbf{y}_{\mathbf{1}}, \mathbf{y}_{\mathbf{2}}, \ldots, \mathbf{y}_{\mathbf{p}}\right\}$. Edge set $W=A$-edges $\cup B$-edges $\cup C$-edges $\cup D$-edges $\cup$ E-edges, where A-edges $=\left\{\left(\mathbf{x}_{\mathbf{j}}, \mathbf{z}_{\mathbf{i}}\right) \mid A^{\lambda}(i, j) \neq 0\right\}$, B-edges $=$ $\left\{\left(\mathbf{u}_{\mathbf{j}}, \mathbf{z}_{\mathbf{i}}\right) \mid B^{\lambda}(i, j) \neq 0\right\}, C$-edges $=\left\{\left(\mathbf{x}_{\mathbf{j}}, \mathbf{y}_{\mathbf{i}}\right) \mid C^{\lambda}(i, j) \neq\right.$ $0\}$, D-edges $=\left\{\left(\mathbf{u}_{\mathbf{j}}, \mathbf{y}_{\mathbf{i}}\right) \mid D^{\lambda}(i, j) \neq 0\right\}$ and E-edges $=$ $\left\{\left(\mathbf{x}_{\mathbf{j}}, \mathbf{z}_{\mathbf{i}}\right) \mid E^{\lambda}(i, j) \neq 0\right\}$. Here, $M^{\lambda}(i, j)$ is the $(i, j)$ th element of matrix $M^{\lambda}$ and $\left(\mathbf{v}_{\mathbf{1}}, \mathbf{v}_{\mathbf{2}}\right)$ denotes a directed edge from vertex $\mathbf{v}_{\mathbf{1}} \in \mathcal{V}$ to vertex $\mathbf{v}_{\mathbf{2}} \in \mathcal{V}$. Note that to differentiate the state, the output and the dynamic variables from their corresponding vertices, the latter are written in bold font. Hereafter, we illustrate the proposed digraph representation with an example.

Example 2 Consider the structured system defined by:

$\begin{aligned} E^{\lambda} & =\left(\begin{array}{ccccc}\lambda_{1} & \lambda_{2} & 0 & 0 & 0 \\ 0 & \lambda_{3} & \lambda_{4} & 0 & 0 \\ 0 & 0 & \lambda_{5} & 0 & 0 \\ 0 & 0 & 0 & \lambda_{6} & \lambda_{7}\end{array}\right), A^{\lambda}=\left(\begin{array}{ccccc}0 & 0 & 0 & \lambda_{8} & \lambda_{9} \\ 0 & 0 & \lambda_{10} & 0 & \lambda_{11} \\ \lambda_{12} & 0 & 0 & 0 & 0 \\ 0 & 0 & 0 & 0 & \lambda_{13}\end{array}\right), B^{\lambda}=\left(\begin{array}{cc}0 & 0 \\ \lambda_{14} & 0 \\ \lambda_{15} & 0 \\ \lambda_{16} & 0\end{array}\right), \\ C^{\lambda} & =\left(\begin{array}{ccccc}\lambda_{17} & \lambda_{18} & 0 & 0 & 0 \\ 0 & 0 & \lambda_{19} & 0 & 0 \\ 0 & 0 & \lambda_{20} & 0 & 0\end{array}\right) \text { and } D^{\lambda}=\left(\begin{array}{cc}0 & 0 \\ 0 & 0 \\ 0 & \lambda_{21}\end{array}\right) . \text { To such a model, we }\end{aligned}$ associate the digraph in Figure 1.

Let us now give some useful definitions and notations.

- Two edges are disjoint if they have no common vertex. 


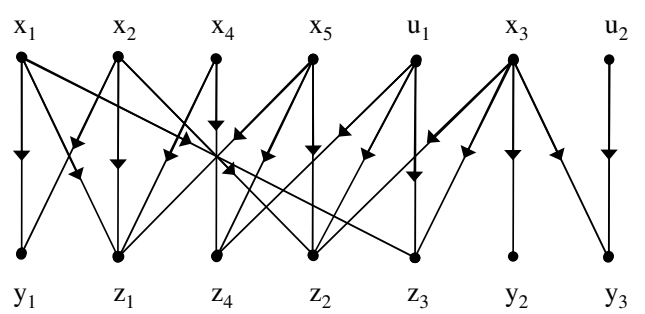

Figure 1. Bipartite graph $B\left(\Sigma_{\Lambda}\right)$ associated to the system of Example 2

In Example 2, edges $\left(\mathbf{u}_{2}, \mathbf{y}_{\mathbf{3}}\right)$ and $\left(\mathbf{x}_{\mathbf{3}}, \mathbf{y}_{2}\right)$ are disjoint but not $\left(\mathbf{u}_{2}, \mathbf{y}_{3}\right)$ and $\left(\mathbf{x}_{3}, \mathbf{y}_{3}\right)$ neither $\left(\mathbf{x}_{3}, \mathbf{y}_{3}\right)$ and $\left(\mathbf{x}_{3}, \mathbf{y}_{2}\right)$.

Let $\mathcal{V}_{1}$ and $\mathcal{V}_{2}$ denote two subsets of $\mathcal{V}$. The cardinality of $\mathcal{V}_{1}$ is noted card $\left(\mathcal{V}_{1}\right)$.

- $\theta\left(\mathcal{V}_{1}, \mathcal{V}_{2}\right)$ is the maximal number of disjoint edges from $\mathcal{V}_{1}$ to $\mathcal{V}_{2}$.

- A matching in a bipartite graph $B\left(\Sigma_{\Lambda}\right)=\left(\mathbf{V}^{+}, \mathbf{V}^{-}, W\right)$ is an edge set $M \subseteq W$ such that all the edges of $M$ are disjoint. A matching is maximal if it has a maximal cardinality which is equal to $\theta\left(\mathbf{V}^{+}, \mathbf{V}^{-}\right)$.

We can associate to each maximal matching $M$, a non bipartite digraph noted $B_{M}\left(\Sigma_{\Lambda}\right)=\left(\mathbf{V}^{+}, \mathbf{V}^{-}, \bar{W}\right)$ where $\left(\mathbf{v}_{\mathbf{1}}, \mathbf{v}_{\mathbf{2}}\right) \in$ $\bar{W} \Leftrightarrow\left(\mathbf{v}_{\mathbf{1}}, \mathbf{v}_{\mathbf{2}}\right) \in W$ or $\left(\mathbf{v}_{\mathbf{2}}, \mathbf{v}_{\mathbf{1}}\right) \in M$. We denote by $\partial^{+} \mathbf{M}$ (resp. $\left.\partial^{-} \mathbf{M}\right)$ the set of vertices in $\mathbf{V}^{+}$(resp. in $\mathbf{V}^{-}$) covered by the edges of $M$. We note $\mathbf{S}_{\mathbf{0}}^{+}=\mathbf{V}^{+} \backslash \partial^{+} \mathbf{M}$ and $\mathbf{S}_{\mathbf{0}}^{-}=\mathbf{V}^{-} \backslash \partial^{-} \mathbf{M}$. In $B_{M}\left(\Sigma_{\Lambda}\right)$, two vertices $\mathbf{v}_{\mathbf{i}}$ and $\mathbf{v}_{\mathbf{j}}$ are said to be strongly connected if it exists a path from $\mathbf{v}_{\mathbf{i}}$ to $\mathbf{v}_{\mathbf{j}}$ and a path from $\mathbf{v}_{\mathbf{j}}$ to $\mathbf{v}_{\mathbf{i}}$. The relation "is strongly connected to" is an equivalence relation and we can define its equivalence classes. We call each equivalent class a strongly connected component. These strongly components are well-known in the graph theory (Murota, 1987). They can be ordered using a partial order relation " $\leqslant$ " defined between two strongly connected components $\mathbf{V}_{\mathbf{i}}$ and $\mathbf{V}_{\mathbf{j}}$ as $\mathbf{V}_{\mathbf{i}} \leqslant \mathbf{V}_{\mathbf{j}}$ if there exists a path from an element of $\mathbf{V}_{\mathbf{j}}$ to an element of $\mathbf{V}_{\mathbf{i}}$.

We can decompose uniquely the obtained bipartite graph into partially ordered irreducible components denoted $B_{i}=$ $\left(\mathbf{V}_{\mathbf{i}}^{+}, \mathbf{V}_{\mathbf{i}}^{-}, \mathbf{W}_{\mathbf{i}}\right)$, using Dulmage-Mendelsohn decomposition (Dulmage and Mendelsohn, 1958; Murota, 1987) described below to make the paper self-contained:

$\leftrightarrow$ Find a maximal matching $M$ in $B\left(\Sigma_{\Lambda}\right)$,

$\leftrightarrow$ Let $\mathbf{V}_{\mathbf{0}}^{+}=\mathbf{S}_{\mathbf{0}}^{+} \cup\left\{\mathbf{v} \in \mathbf{V}^{+}, \exists\right.$ a path in $B_{M}\left(\Sigma_{\Lambda}\right)$ from $\mathbf{S}_{\mathbf{0}}^{+}$to $\left.\mathbf{v}\right\}$

$\leftrightarrow \mathbf{V}_{\mathbf{0}}^{-}=\left\{\mathbf{v} \in \mathbf{V}^{-}, \exists\right.$ a path in $B_{M}\left(\Sigma_{\Lambda}\right)$ from $\mathbf{S}_{\mathbf{0}}^{+}$to $\left.\mathbf{v}\right\}$.

$\rightarrow \mathbf{V}_{\mathbf{0}} \stackrel{\text { def }}{=} \mathbf{V}_{\mathbf{0}}^{-} \cup \mathbf{V}_{\mathbf{0}}^{+}$.

$\rightarrow$ Let $\mathbf{V}_{\infty}^{+}=\left\{\mathbf{v} \in \mathbf{V}^{+}, \exists\right.$ a path in $B_{M}\left(\Sigma_{\Lambda}\right)$ from $\mathbf{v}$ to $\left.\mathbf{S}_{0}^{-}\right\}$

$\leftrightarrow \mathbf{V}_{\infty}^{-}=\mathbf{S}_{\mathbf{0}}^{-} \cup\left\{\mathbf{v} \in \mathbf{V}^{-}, \exists\right.$ a path in $B_{M}\left(\Sigma_{\Lambda}\right)$ from $\mathbf{v}$ to $\left.\mathbf{S}_{\mathbf{0}}^{-}\right\}$.

$\rightarrow \mathbf{V}_{\infty} \stackrel{\text { def }}{=} \mathbf{V}_{\infty}^{-} \cup \mathbf{V}_{\infty}^{+}$.

$\leftrightarrow$ For $i=1, \ldots, r$, let $\mathbf{V}_{\mathbf{i}}$ be the strongly connected component of the graph obtained from $B_{M}\left(\Sigma_{\Lambda}\right)$ after deleting the vertices of $\mathbf{V}_{\mathbf{0}}$ and $\mathbf{V}_{\infty}$.

$\leftrightarrow$ For $i=1, \ldots, r, \mathbf{V}_{\mathbf{i}}^{+}=\mathbf{V}_{\mathbf{i}} \cap \mathbf{V}_{\mathbf{i}}$ and $\mathbf{V}_{\mathbf{i}}^{-}=\mathbf{V}^{-} \cap \mathbf{V}_{\mathbf{i}}$.

It is important to note that the obtained subsets $\mathbf{V}_{\mathbf{i}}^{+}$and $\mathbf{V}_{\mathbf{i}}^{-}$for $i=0,1,2, \ldots, r, \infty$ are the same whatever the choice of the maximal matching $M$ (Dulmage and Mendelsohn, 1958; Murota, 1987). $B_{0}$ is called the minimal inconsistent part and $B_{\infty}$ is the maximal inconsistent part.
In Example 2, since $M$ covers all the vertices of $\mathbf{V}^{-}$ and $\mathbf{V}^{+}$, we have that $\mathbf{V}_{\mathbf{0}}=\mathbf{V}_{\infty}=\emptyset$. Furthermore, we construct $B_{M}\left(\Sigma_{\Lambda}\right)$ by adding the following edges to $B\left(\Sigma_{\Lambda}\right):\left\{\left(\mathbf{y}_{1}, \mathbf{x}_{1}\right),\left(\mathbf{z}_{1}, \mathbf{x}_{\mathbf{2}}\right),\left(\mathbf{z}_{\mathbf{4}}, \mathbf{x}_{\mathbf{4}}\right),\left(\mathbf{z}_{\mathbf{2}}, \mathbf{x}_{\mathbf{5}}\right),\left(\mathbf{z}_{\mathbf{3}}, \mathbf{u}_{\mathbf{1}}\right)\right.$, $\left.\left(\mathbf{y}_{\mathbf{2}}, \mathbf{x}_{\mathbf{3}}\right),\left(\mathbf{y}_{\mathbf{3}}, \mathbf{u}_{2}\right)\right\}$. we have the following decomposition: $\mathbf{V}_{\mathbf{1}}^{+}=\left\{\mathbf{u}_{\mathbf{2}}\right\}, \mathbf{V}_{\mathbf{1}}^{-}=\left\{\mathbf{y}_{\mathbf{3}}\right\}, \mathbf{V}_{\mathbf{2}}^{+}=\left\{\mathbf{x}_{\mathbf{3}}\right\}, \mathbf{V}_{\mathbf{2}}^{-}=\left\{\mathbf{y}_{\mathbf{2}}\right\}, \mathbf{V}_{\mathbf{3}}^{+}=$ $\left\{\mathbf{u}_{1}, \mathbf{x}_{1}, \mathbf{x}_{2}, \mathbf{x}_{4}, \mathbf{x}_{5}\right\}$ and $\mathbf{V}_{\mathbf{3}}^{-}=\left\{\mathbf{y}_{1}, \mathbf{z}_{1}, \mathbf{z}_{2}, \mathbf{z}_{3}, \mathbf{z}_{4}\right\}$.

- We say that component $B_{i}$ contains an $E$-edge if there exists an $E$-edge connecting an element of $\mathbf{V}_{\mathbf{i}}^{+}$to another one of $\mathbf{V}_{\mathbf{i}}^{-}$.

- We define $\bar{B}\left(\Sigma_{\Lambda}\right)=\left(\mathbf{V}^{+}, \mathbf{V}^{-}, \bar{W}\right)$ a bipartite graph as the subgraph of $B\left(\Sigma_{\Lambda}\right)=\left(\mathbf{V}^{+}, \mathbf{V}^{-}, W\right)$ from which we remove the E-edges set i.e. $\mathbf{V}^{+}=\mathbf{X} \cup \mathbf{U}, \mathbf{V}^{-}=\mathbf{Y} \cup \mathbf{Z}$ and $\bar{W}=A$-edges $\cup B$-edges $\cup C$-edges $\cup D$-edges.

- We denote by $\bar{B}_{i}=\left(\overline{\mathbf{V}}_{\mathbf{i}}^{+}, \overline{\mathbf{V}}_{\mathbf{i}}^{-}, \overline{\mathbf{W}}_{\mathbf{i}}\right), i=0,1, \ldots, \bar{r}, \infty$ the irreducible components of $\bar{B}\left(\Sigma_{\Lambda}\right)$ obtained after using Dulmage-Mendelsohn $(D-M)$ decomposition.

For system of Example 2, the results of $D-M$ decomposition are: $\overline{\mathbf{V}}_{\mathbf{0}}=\overline{\mathbf{V}}_{\infty}=\emptyset, \overline{\mathbf{V}}_{1}^{+}=\left\{\mathbf{u}_{\mathbf{2}}\right\}, \overline{\mathbf{V}}_{\mathbf{1}}^{-}=\left\{\mathbf{y}_{\mathbf{3}}\right\}, \overline{\mathbf{V}}_{\mathbf{2}}^{+}=\left\{\mathbf{x}_{\mathbf{3}}\right\}$, $\overline{\mathbf{V}}_{\mathbf{2}}^{-}=\left\{\mathbf{y}_{\mathbf{2}}\right\}, \overline{\mathbf{V}}_{\mathbf{3}}^{+}=\left\{\mathbf{x}_{\mathbf{2}}\right\}, \overline{\mathbf{V}}_{\mathbf{3}}^{-}=\left\{\mathbf{y}_{\mathbf{1}}\right\}, \overline{\mathbf{V}}_{\mathbf{4}}^{+}=\left\{\mathbf{x}_{\mathbf{1}}\right\}, \overline{\mathbf{V}}_{\mathbf{4}}^{-}=\left\{\mathbf{z}_{\mathbf{3}}\right\}$, $\overline{\mathbf{V}}_{5}^{+}=\left\{\mathbf{x}_{4}\right\}, \overline{\mathbf{V}}_{\mathbf{5}}^{-}=\left\{\mathbf{z}_{1}\right\}, \overline{\mathbf{V}}_{\mathbf{6}}^{+}=\left\{\mathbf{u}_{1}, \mathbf{x}_{5}\right\}$ and $\overline{\mathbf{V}}_{\mathbf{6}}^{-}=\left\{\mathbf{z}_{2}, \mathbf{z}_{4}\right\}$.

\section{Main results}

\subsection{Recalls on the state right-hand side observability of} regular descriptor systems without unknown input

Let us denote $P(s)=\left(\begin{array}{cc}A^{\lambda}-s E^{\lambda} & B^{\lambda} \\ C^{\lambda} & D^{\lambda}\end{array}\right)$ the pencil matrix of system $\Sigma_{\Lambda}$. Generic rank of matrix $P(s)$, denoted $g \_\operatorname{rank}(P(s))$ depends on $s$. Hence, $g \_r a n k(P(s))=r, \forall s \in$ $\mathbb{C}$ means that for almost all parameter values $\lambda \in \mathbb{R}^{h}$, $\operatorname{rank}(P(s))=r, \forall s \in \mathbb{C}$.

The generic right-hand side observability of structured system $\Sigma_{\Lambda}$ is equivalent to $g_{-} \operatorname{rank}\left(\begin{array}{cc}A^{\lambda}-s E^{\lambda} & B^{\lambda} \\ C^{\lambda} & D^{\lambda}\end{array}\right)=n+m$, $\forall s \in \mathbb{C}$, (Hou and Müller, 1999a).

To establish the state and input right-hand side observability for the most general descriptor systems, we first recall the graphical results established in (Murota, 1987) for regular descriptor systems without unknown input:

$$
\Sigma_{\Lambda}^{R}:\left\{\begin{array}{r}
E^{\lambda} \dot{x}=A^{\lambda} x \\
y=C^{\lambda} x \\
z \stackrel{\text { def }}{=} E^{\lambda} x
\end{array}\right.
$$

where $E^{\lambda} \in \mathbb{R}^{n \times n}$ and $A^{\lambda} \in \mathbb{R}^{n \times n}$, with $\operatorname{det}\left(A^{\lambda}-s E^{\lambda}\right) \not \equiv 0$. Then, we extend the obtained results to handle the state and input right-hand side observability of any descriptor system. The following result is given in (Murota, 1987):

Theorem 3 Structured system $\Sigma_{\Lambda}^{R}$ is generically right-hand side observable or equivalently g_rank $\left(\begin{array}{c}A^{\lambda}-s E^{\lambda} \\ C^{\lambda}\end{array}\right)=n$ iff the two following conditions are satisfied:

Cond.1) after the D-M decomposition of $\bar{B}\left(\Sigma_{\Lambda}\right), \overline{\mathbf{V}}_{\mathbf{0}}=\emptyset$, Cond.2) after the $D-M$ decomposition of $B\left(\Sigma_{\Lambda}\right), \forall i=$ $1, \ldots, r$, component $B_{i}$ does not contain any E-edge i.e. there is no E-edge linking $\mathbf{V}_{\mathbf{i}}^{+}$to $\mathbf{V}_{\mathbf{i}}^{-}$. 
4.2 Input and state right-hand side observability of descriptor systems

Consider now the more general case of structured descriptor systems with unknown inputs $\Sigma_{\Lambda}$ defined by Equations (1). The generic right-hand side observability of structured system $\Sigma_{\Lambda}$ is equivalent to

$$
g \_r a n k\left(\begin{array}{cc}
A^{\lambda}-s E^{\lambda} & B^{\lambda} \\
C^{\lambda} & D^{\lambda}
\end{array}\right)=n+m, \forall s \in \mathbb{C}
$$

Therefore, a necessary graphical condition to the generic right-hand side observability is that in bipartite graph $B\left(\Sigma_{\Lambda}\right)$ associated to $\Sigma_{\Lambda}$, we have $\mathbf{V}_{\mathbf{0}}=\emptyset$ or equivalently, $\theta(\mathbf{X} \cup$ $\mathbf{U}, \mathbf{Z} \cup \mathbf{Y})=n+m$. Indeed, this condition is necessary to have in $\bar{B}\left(\Sigma_{\Lambda}\right), \overline{\mathbf{V}}_{\mathbf{0}}=\emptyset$ which is equivalent to $g_{-} \operatorname{rank}\left(\begin{array}{ll}A^{\lambda} & B^{\lambda} \\ C^{\lambda} & D^{\lambda}\end{array}\right)=$ $n+m$ i.e. that condition (3) is satisfied for $s=0$.

When $\mathbf{V}_{\mathbf{0}}=\emptyset$, then, since by assumption $g_{-} \operatorname{rank}\left(E^{\lambda}\right)=q$, there exists necessarily a complete matching in $B\left(\Sigma_{\Lambda}\right)$ including all the $E$-edges. In this case, this complete matching includes necessarily $n+m-q$ edges associated to the submatrix $\left(\begin{array}{ll}C^{\lambda} & D^{\lambda}\end{array}\right)$. After some row permutations, we can decompose matrix $\left(\begin{array}{ll}C^{\lambda} & D^{\lambda}\end{array}\right)$ in order to obtain $\left(\begin{array}{ll}C_{S}^{\lambda} & D_{S}^{\lambda} \\ C_{r}^{\lambda} & D_{r}^{\lambda}\end{array}\right)$ such that, in the associated bipartite graph, a complete matching covers exactly the edges associated to the square ma$\operatorname{trix}\left(\begin{array}{cc}A^{\lambda}-s E^{\lambda} & B^{\lambda} \\ C_{s}^{\lambda} & D_{s}^{\lambda}\end{array}\right)$. Let us denote $\bar{E}^{\lambda}=\left(\begin{array}{cc}E^{\lambda} & 0 \\ 0 & 0\end{array}\right), \bar{A}^{\lambda}=$ $\left(\begin{array}{ll}A^{\lambda} & B^{\lambda} \\ C_{s}^{\lambda} & D_{s}^{\lambda}\end{array}\right)$ and $\bar{C}^{\lambda}=\left(\begin{array}{ll}C_{r}^{\lambda} & D_{r}^{\lambda}\end{array}\right)$. The obtained system is then square and regular, from (van der Woude, 2002) (Theorem 6 ), because there exists a complete matching in the bipartite graph associated to $\bar{A}^{\lambda}-s \bar{E}^{\lambda}$. Note that the $\bar{E}$-edges set is exactly equal to the original E-edges set. All the hypotheses or usability conditions of Theorem 3 are satisfied and we can then establish:

Proposition 4 Descriptor structured system with unknown inputs $\Sigma_{\Lambda}$ is generically input and state right-hand side observable or equivalently g_rank $\left(\begin{array}{cc}A^{\lambda}-s E^{\lambda} & B^{\lambda} \\ C^{\lambda} & D^{\lambda}\end{array}\right)=n+m$ iff the two following conditions are satisfied:

Cond.1) after the D-M decomposition of $\bar{B}\left(\Sigma_{\Lambda}\right), \overline{\mathbf{V}}_{\mathbf{0}}=\emptyset$, or equivalently $\theta(\mathbf{X} \cup \mathbf{U}, \mathbf{Z} \cup \mathbf{Y})=n+m$ in $\bar{B}\left(\Sigma_{\Lambda}\right)$ (Complete matching condition)

Cond.2) after the $D-M$ decomposition of $B\left(\Sigma_{\Lambda}\right), \forall i=$ $1, \ldots, r$, component $B_{i}$ does not contain any $E$-edge i.e. there is no E-edge linking $\mathbf{V}_{\mathbf{i}}^{+}$to $\mathbf{V}_{\mathbf{i}}^{-}$(s-condition).

In Example 2, condition Cond.1 of Proposition 4 is satisfied but not Cond.2. Indeed, even if between $\mathbf{V}_{1}^{+}$and $\mathbf{V}_{\mathbf{1}}^{-}$as between $\mathbf{V}_{2}^{+}$and $\mathbf{V}_{2}^{-}$there is no $E$-edge, there are edges between $\mathbf{V}_{\mathbf{3}}^{+}$and $\mathbf{V}_{\mathbf{3}}^{-}\left(\left(\mathbf{x}_{1}, \mathbf{z}_{1}\right)\right.$ or $\left(\mathbf{x}_{2}, \mathbf{z}_{1}\right)$ for instance).

\subsection{Input and state right-hand side observability recover- ing by additional sensor implementation}

When the conditions of Proposition 4 are not satisfied, our proposed procedure to recover the input and state right-hand side observability consists of two steps which correspond one by one to the conditions of Proposition 4. In the sequel, we define a new output vector $\Upsilon$ representing the additional sensors collecting the new measurements $\Upsilon(t)=H_{x}^{\lambda} x(t)+$ $H_{u}^{\lambda} u(t)$. Hence, we denote the completed system by $\Sigma_{\Lambda}^{c}$ :

$$
\Sigma_{\Lambda}^{c}:\left\{\begin{aligned}
E^{\lambda} \dot{x}(t) & =A^{\lambda} x(t)+B^{\lambda} u(t) \\
y(t) & =C^{\lambda} x(t)+D^{\lambda} u(t) \\
\Upsilon(t) & =H_{x}^{\lambda} x(t)+H_{u}^{\lambda} u(t)
\end{aligned}\right.
$$

The additional sensor components can be represented by vertex set $\boldsymbol{\Upsilon}$ and edge subsets $H_{x}$-edges and $H_{u}$-edges from respectively $\mathbf{X}$ to $\boldsymbol{\Upsilon}$ and $\mathbf{U}$ to $\boldsymbol{\Upsilon}$. We subdivide vertex subset $\boldsymbol{\Upsilon}$ into two subsets denoted $\boldsymbol{\Upsilon}_{\mathbf{1}}$ and $\boldsymbol{\Upsilon}_{\mathbf{2}}$. Each subset $\boldsymbol{\Upsilon}_{\mathbf{i}}$, $i=1,2$ corresponds to the sensors possibly added at the $i^{\text {th }}$ stage of our procedure.

\subsubsection{Complete matching condition recovering}

First of all, note that the complete matching condition is also equivalent to have in $\bar{B}\left(\Sigma_{\Lambda}\right)$

$$
\forall \mathbf{\Omega} \subseteq \mathbf{X} \cup \mathbf{U}, \theta(\mathbf{\Omega}, \mathbf{Z} \cup \mathbf{Y})=\operatorname{card}(\mathbf{\Omega})
$$

We say that there is a dilation in the digraph of the system when the latter condition is not satisfied for some vertex subset $\mathbf{\Omega} \subseteq \mathbf{X} \cup \mathbf{U}$. The aim of additional sensors at this first stage is to eliminate all the dilations. Then, the fact that condition Cond.1 of Proposition 4 is not satisfied i.e. $\theta(\mathbf{X} \cup \mathbf{U}, \mathbf{Z} \cup \mathbf{Y})<$ $\operatorname{card}(\mathbf{X})+\operatorname{card}(\mathbf{U})=n+m=\operatorname{card}\left(\mathbf{V}^{+}\right)$in $\bar{B}\left(\Sigma_{\Lambda}\right)$ or in other words $\theta\left(\mathbf{V}^{+}, \mathbf{V}^{-}\right)<\operatorname{card}\left(\mathbf{V}^{+}\right)$in $\bar{B}\left(\Sigma_{\Lambda}\right)$, implies that some additional sensor vertices are needed to complete $\mathbf{V}^{-}$. This number is at least equal to $\operatorname{card}\left(\overline{\mathbf{V}}_{\mathbf{0}}^{+}\right)-\operatorname{card}\left(\overline{\mathbf{V}}_{\mathbf{0}}^{-}\right)$. In addition to the number of required sensors, another problem is to precise as finely as possible their location because obviously adding anywhere $\operatorname{card}\left(\overline{\mathbf{V}}_{\mathbf{0}}^{+}\right)-\operatorname{card}\left(\overline{\mathbf{V}}_{\mathbf{0}}^{-}\right)$sensors does not allow to recover the complete matching condition. Using the $D-M$ decomposition of the bipartite graph $\bar{B}\left(\Sigma_{\Lambda}\right)$, we can state:

Proposition 5 Consider structured linear system $\Sigma_{\Lambda}$ represented by bipartite graph $\bar{B}\left(\Sigma_{\Lambda}\right)$. To recover the complete matching condition, the minimal number of additional sensors, noted $\Upsilon_{\mathbf{1}}$ in bipartite graph $\bar{B}\left(\Sigma_{\Lambda}^{c}\right)$, is equal to $\gamma=n+m-\theta\left(\mathbf{V}^{+}, \mathbf{V}^{-}\right)=\operatorname{card}\left(\overline{\mathbf{V}}_{\mathbf{0}}^{+}\right)-\operatorname{card}\left(\overline{\mathbf{V}}_{\mathbf{0}}^{-}\right)$. These additional sensors must measure $\gamma$ states and unknown inputs in $\overline{\mathbf{V}}_{\mathbf{0}}^{+}$such that we obtain a maximal matching of size $n+m$ in $\bar{B}\left(\Sigma_{\Lambda}^{c}\right)$ i.e. $\theta\left(\mathbf{X} \cup \mathbf{U}, \mathbf{Z} \cup \mathbf{Y} \cup \mathbf{\Upsilon}_{\mathbf{1}}\right)=n+m$ in $\bar{B}\left(\Sigma_{\Lambda}^{c}\right)$.

\section{Proof:}

Sufficiency: The Dulmage-Mendelsohn decomposition characterizes all the maximal matchings (Murota, 1987) and so if $\operatorname{card}\left(\overline{\mathbf{V}}_{\mathbf{0}}^{+}\right)-\operatorname{card}\left(\overline{\mathbf{V}}_{\mathbf{0}}^{-}\right)$edges are added between $\overline{\mathbf{S}}_{\mathbf{0}}^{+}$and the additional sensors, in order to form a matching of size $\operatorname{card}\left(\overline{\mathbf{V}}_{\mathbf{0}}^{+}\right)-\operatorname{card}\left(\overline{\mathbf{V}}_{\mathbf{0}}^{-}\right)$, then in $\bar{B}\left(\Sigma_{\Lambda}^{c}\right), \overline{\mathbf{S}}_{\mathbf{0}}^{+}=\emptyset$. Thus, there is no minimal inconsistent part $\overline{\mathbf{B}}_{\mathbf{0}}$ in $\bar{B}\left(\Sigma_{\Lambda}^{c}\right)$. In this case, we obtain a maximal matching of size $n+m$ between $\mathbf{V}^{+}$and $\mathbf{Z} \cup \mathbf{Y} \cup \Upsilon_{\mathbf{1}}$ in $\bar{B}\left(\Sigma_{\Lambda}^{c}\right)$.

Necessity: Let us consider a solution which provides a maximal matching $M^{c}$ of size $n+m$ in $\bar{B}\left(\Sigma_{\Lambda}^{c}\right) . M^{c}$ covers all the state and input vertices of $\mathbf{V}^{+}$and in particular the elements of $\overline{\mathbf{V}}_{\mathbf{0}}^{+}$. Thus, there are at least $\operatorname{card}\left(\overline{\mathbf{V}}_{\mathbf{0}}^{+}\right)-\operatorname{card}\left(\overline{\mathbf{V}}_{\mathbf{0}}^{-}\right)$edges 
from $\overline{\mathbf{V}}_{\mathbf{0}}^{+}$to the additional sensors.

It can be useful to specify more precisely the additional sensor location to recover the complete matching condition. At this aim, let us define by $\bar{B}_{N}\left(\Sigma_{\Lambda}\right)$ the non-directed graph corresponding to $\bar{B}\left(\Sigma_{\Lambda}\right)$. We call $\overline{\mathbf{V}}_{\mathbf{0}}$-path every simple path of $\bar{B}_{N}\left(\Sigma_{\Lambda}\right)$ which covers only vertices of $\overline{\mathbf{V}}_{\mathbf{0}}$. For each $\overline{\mathbf{V}}_{\mathbf{0}}$-path $P$, we define a vertex subset $\vartheta(P)$ such that $\vartheta(P)=\left\{\mathbf{v} \in \overline{\mathbf{V}}_{\mathbf{0}}^{+}\right.$, such that $P$ covers $\left.\mathbf{v}\right\}$. Finally, we say that a $\overline{\mathbf{V}}_{\mathbf{0}}$-path $P$ is maximal, if there does not exist a $\overline{\mathbf{V}}_{\mathbf{0}}$-path $P^{\prime}$ such that $\vartheta(P) \subset \vartheta\left(P^{\prime}\right)$. It is quite easy to see that to recover the complete matching condition, it is necessary to have in $\bar{B}\left(\Sigma_{\Lambda}^{C}\right)$ :

$$
\text { for each maximal } \overline{\mathbf{V}}_{\mathbf{0}} \text {-path } P, \theta\left(\vartheta(P), \boldsymbol{\Upsilon}_{\mathbf{1}}\right) \neq 0
$$

It is also possible to prove that, to recover the complete matching condition, it is necessary to have, in $\bar{B}\left(\Sigma_{\Lambda}^{c}\right)$, for each maximal $\overline{\mathbf{V}}_{\mathbf{0}}$-path $P$ :

$$
\theta\left(\overline{\mathbf{V}}_{\mathbf{0}}^{+}, \boldsymbol{\Upsilon}_{\mathbf{1}} \cup \overline{\mathbf{V}}_{\mathbf{0}}^{-}\right)-\theta\left(\overline{\mathbf{V}}_{\mathbf{0}}^{+} \backslash \vartheta(P), \boldsymbol{\Upsilon}_{\mathbf{1}} \cup \overline{\mathbf{V}}_{\mathbf{0}}^{-}\right)>0
$$

Conditions (5) and (6) ensure that there is at least one sensor dedicated to take a measurement in each subset $\vartheta(P)$. In fact, these above conditions can be seen as complementary tools which allow to have effortlessly a better precision on the sensor location, since we obtain a kind of repartition of the required $\gamma$ sensors.

Example 6 Consider the structured linear descriptor system represented by the bipartite graph depicted in Figure 2, where E-edges set is $\left\{\left(\mathbf{x}_{1}, \mathbf{z}_{1}\right),\left(\mathbf{x}_{1}, \mathbf{z}_{4}\right),\left(\mathbf{x}_{\mathbf{2}}, \mathbf{z}_{2}\right),\left(\mathbf{x}_{\mathbf{3}}, \mathbf{z}_{3}\right),\left(\mathbf{x}_{\mathbf{4}}, \mathbf{z}_{\mathbf{4}}\right),\left(\mathbf{x}_{5}, \mathbf{z}_{4}\right)\right\} \quad$ and A-edges set is $\left\{\left(\mathbf{x}_{2}, \mathbf{z}_{1}\right),\left(\mathbf{x}_{\mathbf{4}}, \mathbf{z}_{1}\right),\left(\mathbf{x}_{3}, \mathbf{z}_{2}\right),\left(\mathbf{x}_{3}, \mathbf{z}_{3}\right),\left(\mathbf{x}_{1}, \mathbf{z}_{\mathbf{4}}\right)\right.$, $\left.\left(\mathbf{x}_{\mathbf{4}}, \mathbf{z}_{\mathbf{4}}\right),\left(\mathbf{x}_{\mathbf{5}}, \mathbf{z}_{\mathbf{4}}\right)\right\}$.

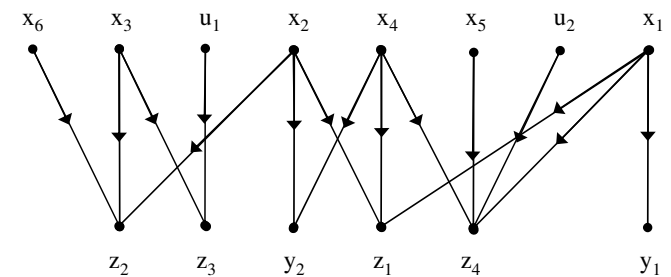

Figure 2. Bipartite graph $B\left(\Sigma_{\Lambda}\right)$ representing system of Example 6

We have the following decomposition in $B\left(\Sigma_{\Lambda}\right)$ : $\overline{\mathbf{V}}_{\mathbf{0}}^{+}=\left\{\mathbf{u}_{\mathbf{1}}, \mathbf{x}_{\mathbf{3}}, \mathbf{x}_{\mathbf{6}}, \mathbf{u}_{\mathbf{2}}, \mathbf{x}_{\mathbf{5}}\right\}, \quad \overline{\mathbf{V}}_{\mathbf{0}}^{-}=\left\{\mathbf{z}_{\mathbf{2}}, \mathbf{z}_{\mathbf{3}}, \mathbf{z}_{\mathbf{4}}\right\}$, $\overline{\mathbf{V}}_{\infty}^{+}=\overline{\mathbf{V}}_{\infty}^{-}=\emptyset, \overline{\mathbf{V}}_{1}^{+}=\left\{\mathbf{x}_{\mathbf{1}}\right\}, \overline{\mathbf{V}}_{\mathbf{1}}^{-}=\left\{\mathbf{y}_{\mathbf{1}}\right\}, \overline{\mathbf{V}}_{\mathbf{2}}^{+}=\left\{\mathbf{x}_{\mathbf{2}}, \mathbf{x}_{\mathbf{4}}\right\}$ and $\overline{\mathbf{V}}_{\mathbf{2}}^{-}=\left\{\mathbf{y}_{\mathbf{2}}, \mathbf{z}_{\mathbf{1}}\right\}$. Furthermore, in $\bar{B}\left(\Sigma_{\Lambda}\right)$, we have that $\overline{\mathbf{V}}_{\mathbf{0}}^{+}=\left\{\mathbf{x}_{\mathbf{3}}, \mathbf{x}_{\mathbf{6}}, \mathbf{u}_{\mathbf{2}}, \mathbf{x}_{\mathbf{5}}\right\}, \overline{\mathbf{V}}_{\mathbf{0}}^{-}=\left\{\mathbf{z}_{\mathbf{2}}, \mathbf{z}_{\mathbf{4}}\right\}$. As $\overline{\mathbf{V}}_{\mathbf{0}}^{+} \neq \emptyset$, condition Cond.1 of Proposition 4 is not satisfied. The number of necessary sensors to recover this condition is $\operatorname{card}\left(\overline{\mathbf{V}}_{\mathbf{0}}^{+}\right)-\operatorname{card}\left(\overline{\mathbf{V}}_{\mathbf{0}}^{-}\right)=2$ and they must take their measurements in $\left\{\mathbf{x}_{\mathbf{3}}, \mathbf{x}_{\mathbf{6}}, \mathbf{u}_{\mathbf{2}}, \mathbf{x}_{\mathbf{5}}\right\}$. However, if we add two sensors such that the first one measures $x_{3}$ and the second one $x_{6}$, condition Cond.1 of Proposition 4 will not be satisfied. If we compute the maximal $\overline{\mathbf{V}}_{\mathbf{0}}$-paths, we have : $P_{1}=\mathbf{x}_{\mathbf{6}} \rightarrow \mathbf{z}_{2} \rightarrow \mathbf{x}_{3}$ and $P_{2}=\mathbf{u}_{2} \rightarrow \mathbf{z}_{\mathbf{4}} \rightarrow \mathbf{x}_{\mathbf{5}}$. Therefore, the set of additional sensors $\Upsilon_{1}$ must satisfy:

$$
\left\{\begin{array}{l}
\theta\left(\left\{\mathbf{x}_{\mathbf{3}}, \mathbf{x}_{\mathbf{6}}, \mathbf{u}_{\mathbf{2}}, \mathbf{x}_{\mathbf{5}}\right\}, \boldsymbol{\Upsilon}_{\mathbf{1}}\right) \geq 2 \\
\theta\left(\left\{\mathbf{x}_{\mathbf{3}}, \mathbf{x}_{\mathbf{6}}\right\}, \boldsymbol{\Upsilon}_{\mathbf{1}}\right) \geq 1 \\
\theta\left(\left\{\mathbf{u}_{\mathbf{2}}, \mathbf{x}_{\mathbf{5}}\right\}, \boldsymbol{\Upsilon}_{\mathbf{1}}\right) \geq 1
\end{array}\right.
$$

In the case of Example 6, condition (6) is satisfied if the above constraints are also satisfied.

\subsection{2 s-condition recovering}

Assume that condition Cond.1 of Proposition 4 is verified by the system. If it is not the case, then we must add first some sensors as it is described in the previous subsection before dealing with the $s$-condition recovering stage. We denote by $\tilde{\mathbf{Y}}=\mathbf{Y} \cup \boldsymbol{\Upsilon}_{\mathbf{1}}$ the output vertex subset at the beginning of this second and last stage of our procedure.

On the one hand, if condition Cond.1 is satisfied then $\overline{\mathbf{V}}_{\mathbf{0}}=\emptyset$ and so $\mathbf{V}_{\mathbf{0}}=\emptyset$. Thus, adding a sensor on $\mathbf{V}_{\mathbf{i}}, i=1, \ldots, r$, makes that this strongly connected component disappears and all its vertices will belong to $\mathbf{V}_{\infty}$. On the other hand, if condition Cond.1 is satisfied, when two vertices belong to the same strongly connected component, then, after adding a new sensor anywhere in the bipartite graph, they remain in the same strongly connected component. Therefore, the only way to satisfy Cond.2 of Proposition 4 is to add some sensors in order to make that all the vertices of strongly connected components $\mathbf{B}_{\mathbf{i}}, i=1, \ldots, r$, which contain $E$ edges, will belong to $\mathbf{B}_{\infty}$ in completed system $B\left(\Sigma_{\Lambda}^{c}\right)$. This reasoning is summarized in the following proposition:

Proposition 7 Consider structured linear system $\Sigma_{\Lambda}$ represented by bipartite graph $B\left(\Sigma_{\Lambda}\right)$. Assume that the complete matching condition is satisfied i.e. $\overline{\mathbf{V}}_{\mathbf{0}}=\emptyset$. To recover the s-condition, the additional sensors $\Upsilon_{2}$ must measure at least one state in each subset $\mathbf{V}_{\mathbf{i}}^{+}, i=1, \ldots, r$, if $B_{i}$ contains an E-edge i.e. for $i=1, \ldots, r$, if $B_{i}$ contains an E-edge then we must have $\theta\left(\mathbf{V}_{\mathbf{i}}^{+}, \mathbf{\Upsilon}_{\mathbf{2}}\right) \neq 0$

Note that it is possible that only one sensor, which takes its measurement in each strongly connected component containing an $E$-edge allows to satisfy the requirements of the $s$-condition.

From a global point of view, the minimal number of additional sensors, required to recover both the complete matching condition and the $s$-condition, is then less or equal to $\gamma+1$, where $\gamma=\operatorname{card}\left(\overline{\mathbf{V}}_{\mathbf{0}}^{+}\right)-\operatorname{card}\left(\overline{\mathbf{V}}_{\mathbf{0}}^{-}\right)=n+m-\theta(\mathbf{X} \cup \mathbf{U}, \mathbf{Y} \cup \mathbf{Z})$, and $\theta(\mathbf{X} \cup \mathbf{U}, \mathbf{Y} \cup \mathbf{Z})$ is computed here in $\bar{B}\left(\Sigma_{\Lambda}\right)$ (i.e. without $E$-edges). In fact, we have two cases, if $\mathbf{V}_{\infty}^{+}$includes an element of $\overline{\mathbf{V}}_{\mathbf{0}}^{+}$then the minimal number of necessary sensors is $\gamma$. In this first case, we can use a sensor to recover both complete matching condition by putting it on an element of $\mathbf{V}_{\infty}^{+} \cap \overline{\mathbf{V}}_{\mathbf{0}}^{+}$and satisfying the additional constraint of Proposition 7. Otherwise, if $\mathbf{V}_{\infty}^{+} \cap \overline{\mathbf{V}}_{\mathbf{0}}^{+}=\emptyset$, the sensors used in the first stage allow just to transform set $\overline{\mathbf{V}}_{\mathbf{0}}$ into several sets $\mathbf{V}_{\mathbf{i}}$, $i \neq 0$ and $i \neq \infty$. Then, these sensors cannot be useful to recover the $s$-condition which goal is to transform some $\mathbf{V}_{\mathbf{i}}$ sets $(i \neq 0$ and $i \neq \infty)$ into a part of $\mathbf{V}_{\infty}$. Therefore, we need a new sensor to recover $s$-condition.

For Example 2, a sensor measuring any component in $\left\{\mathbf{u}_{\mathbf{1}}, \mathbf{x}_{\mathbf{1}}, \mathbf{x}_{\mathbf{2}}, \mathbf{x}_{\mathbf{4}}, \mathbf{x}_{\mathbf{5}}\right\}$ is necessary and sufficient to recover 
the $s$-condition. in this case, $\mathbf{V}_{\mathbf{3}}^{+}=\mathbf{V}_{\mathbf{3}}^{-}=\emptyset, \mathbf{V}_{\infty}^{+}=$

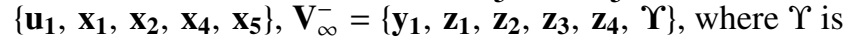
the added sensor such that $\theta\left(\left\{\mathbf{u}_{1}, \mathbf{x}_{\mathbf{1}}, \mathbf{x}_{\mathbf{2}}, \mathbf{x}_{\mathbf{4}}, \mathbf{x}_{\mathbf{5}}\right\}, \boldsymbol{\Upsilon}\right) \neq 0$.

In the case of system described in Example 6, let us assume that to satisfy the complete matching condition, we have added in the first stage of our procedure two sensors measuring respectively $x_{6}$ and $u_{2}$ denoted respectively $\Upsilon_{1}^{1}$ and $\Upsilon_{1}^{2}$. In this case, $\mathbf{V}_{\mathbf{0}}$ will be an empty set and $\mathbf{V}_{\infty}$ remains empty. The Dulmage-Mendelsohn decomposition gives the following components: $\mathbf{V}_{\mathbf{0}}^{+}=\mathbf{V}_{\mathbf{0}}^{-}=\mathbf{V}_{\infty}^{+}=\mathbf{V}_{\infty}^{-}=\emptyset, \mathbf{V}_{\mathbf{1}}^{+}=\left\{\mathbf{x}_{\mathbf{1}}\right\}$, $\mathbf{V}_{\mathbf{1}}^{-}=\left\{\mathbf{y}_{\mathbf{1}}\right\}, \mathbf{V}_{\mathbf{2}}^{+}=\left\{\mathbf{x}_{\mathbf{2}}, \mathbf{x}_{\mathbf{4}}\right\}, \mathbf{V}_{\mathbf{2}}^{-}=\left\{\mathbf{y}_{\mathbf{2}}, \mathbf{z}_{1}\right\}, \mathbf{V}_{\mathbf{3}}^{+}=\left\{\mathbf{u}_{\mathbf{1}}, \mathbf{x}_{\mathbf{3}}, \mathbf{x}_{\mathbf{6}}\right\}$, $\mathbf{V}_{\mathbf{3}}^{-}=\left\{\mathbf{z}_{2}, \mathbf{z}_{\mathbf{3}}, \Upsilon_{\mathbf{1}}^{\mathbf{1}}\right\}, \mathbf{V}_{\mathbf{4}}^{+}=\left\{\mathbf{u}_{2}, \mathbf{x}_{5}\right\}$ and $\mathbf{V}_{\mathbf{4}}^{-}=\left\{\boldsymbol{\Upsilon}_{1}^{\mathbf{2}}, \mathbf{z}_{\mathbf{4}}\right\}$. There are $E$-edges between $\mathbf{V}_{\mathbf{3}}^{+}$and $\mathbf{V}_{\mathbf{3}}^{-}$and between $\mathbf{V}_{4}^{+}$ and $\mathbf{V}_{\mathbf{4}}^{-}$and so $B_{3}$ and $B_{4}$ contains $E$-edges. Therefore, the additional sensor $\Upsilon_{2}$, must satisfy $\theta\left(\left\{\mathbf{u}_{1}, \mathbf{x}_{3}, \mathbf{x}_{\mathbf{6}}\right\}, \Upsilon_{2}\right)=1$ and $\theta\left(\left\{\mathbf{u}_{\mathbf{2}}, \mathbf{x}_{\mathbf{5}}\right\}, \boldsymbol{\Upsilon}_{\mathbf{2}}\right)=1$. For this example, we can take $\Upsilon_{2}=\alpha_{1} x_{3}+\alpha_{2} x_{5}$.

\subsection{Complexity analysis}

From a computational point of view, our proposed approach needs few information about the system and is quite easy to check by means of well-known combinatorial techniques or simply by hand for small systems. More precisely, let us denote by $M$ the number of edges in the digraph and $N=n+m+q+p$ the number of vertices of our graphs. The first step of our procedure requires a Dulmage-Mendelsohn decomposition which can be implemented using an algorithm with a complexity order $O\left(M^{2}\right)=O\left(n^{4}\right)$ (Lovasz and Plummer, 1986; Chen and Kanj, 2003), assuming without loss of generality that $n \geq p$ and $n \geq m$. Next, our procedure requires the calculation of the strongly connected components which can be done using an algorithm which complexity order equals $O(N \log (N))=O(n \log (n))$ (Fleischer $e t$ al., 2000). Checking the observability conditions necessitates only the knowledge of $\mathbf{V}_{\mathbf{0}}$ issued from the DulmageMendelsohn decomposition and at most $n \times q$ tests to know if each $E$-edge belongs to a strongly connected component. This can be done with a $O\left(n^{3}\right)$ complexity order algorithm. The additional sensor location characterization, does not necessitate more complex computation and the proposed method can be implemented using a global algorithm with a polynomial complexity equal to $O\left(n^{4}\right)$.

\section{Conclusion}

In this paper, we propose a graph-theoretic tool to analyze, at first, the state and input generic right-hand side observability of structured linear descriptor systems. The studied system can be underdetermined, overdetermined or square and possibly non-regular. Necessary and sufficient conditions for state and unknown input generic observability are expressed in graphic terms. These conditions extend the ones of (Murota, 1987) which deal with regular descriptor systems without input. Next, when these observability conditions are not satisfied, we study the minimal number of the required additional sensors and either their location or necessary and sufficient conditions to be satisfied by any acceptable location in order to recover the generic state and input observability. It is shown also that our approach uses classical programming techniques and is free from numerical dif- ficulties since it uses well-known combinatorial techniques. Indeed, all the algorithms used in the paper have polynomial complexity orders. This makes our proposed method well-suited to large scale systems.

\section{References}

Aplevich, J. D. (1991). Implicit Linear Systems. Springer. New York.

Boukhobza, T., Hamelin, F. \& Sauter, D. (2006). Observability of structured linear systems in descriptor form: a graph-theoretic approach. Automatica, 42(6), 629-635.

Chen, J. \& Kanj, I. A. (2003). Constrained minimum vertex cover in bipartite graphs: Complexity and parameterized algorithms. Journal of Computer and System Sciences, 67(4), 833-847.

Dai, L. (1989). Singular Control Systems. Springer-Verlag. Berlin.

Dion, J-M., Commault, C., \& Van der Woude, J. (2003). Generic properties and control of linear structured systems: A survey. Automatica, 39(7), 1125-1144

Dulmage, A. L. \& Mendelsohn, N. S. (1958). Coverings of bipartite graphs. Canadian Journal of Mathematics, 10 517-534.

Fleischer, L. K., Hendrickson, B., \& Pinar, A. (2000). On Identifying Strongly Connected Components in Parallel. Lecture Notes in Computer Science. Springer Berlin / Heidelberg.

Geerts, T. (1993). Solvability conditions, consistency and weak consistency for linear differential-algebraic conditions and timeinvariant singular systems: The general case. Linear Algebra Applications, 181, 111-130.

Hou, M., \& Müller, P. C. (1999a). Causal observability of descriptor systems. IEEE Transactions on Automatic Control, 44(1), 158-163.

Hou, M., \& Müller, P. C. (1999b). Observer design for descriptor systems. IEEE Transactions on Automatic Control, 44(1), 164-169.

Hou, M., \& Patton, R. J. (1998). Input observability and input reconstruction. Automatica, 34(6), 789-794.

Lewis, F. L. (1992). A tutorial on the geometric analysis of linear timeinvariant implicit systems. Automatica, 28(1), 119-137.

Lovasz, L. \& Plummer, M. D. (1986). Matching Theory. Annals of Discrete Mathematics, 29, North-Holland, Amsterdam, Netherlands.

Luenberger, D. G. (1977). ADynamic equation in descriptor form. IEEE Transactions on Automatic Control, AC-23(3), 312-321.

Müller, P. C. (2000). Descriptor systems: Pros and cons of system modelling by differential-algebraic equations. Mathematics and Computers in Simulation, 53(4-6), 273-279.

Müller, P. C., \& Hou, M. (1993). On the observer design for descriptor systems. IEEE Transactions on Automatic Control, 38(11), 16661671 .

Murota, K. (1987). System Analysis by Graphs and Matroids. SpringerVerlag. New York, U.S.A.

Nikoukah, R., Campbell, S. L., \& Delebecque, F. (1998). Observer design for general linear time-invariant systems. Automatica, 34(5), 575583.

Reinschke, K. J. (1988). Multivariable Control. A Graph Theoretic Approach.. Springer-Verlag. New York, U.S.A.

Reinschke, K. J., \& Wiedemann, G. (1997). Digraph characterization of structural controllability for linear descriptor systems. Linear Algebra and its Applications, 266, 199-217.

Verghese, G. C., Levy, B. C., \& Kailath, T. (1981). A generalized statespace for singular systems. IEEE Transactions on Automatic Control, AC-26(4), 811-831.

Wiedemann, G. (1999). Strukturelle Zugänge Zur Analyse und Synthese Linearer Regelungssysteme in Deskriptorform. shaker verlag ed.. Shaker Verlag. Aaachen, Germany.

van der Woude, J. (2002). The generic canonical form of a regular structured matrix pencil. Linear Algebra and its Applications, 353(13), 267-288.

Yip, E. L., \& Sincovec, R. F. (1981). Solvability, controllability and observability of continuous descriptor systems. IEEE Transactions on Automatic Control, AC-26(3), 702-707. 